\title{
Direction of Arrival Estimation via ESPRIT Algorithm for Smart Antenna System
}

\author{
Deven Pradhan \\ Dept. of Electronics and Communication Sikkim \\ Manipal Institute of Technology
}

\begin{abstract}
Spectrum sensing is the main aspect of Cognitive Radio. There are several spectrum sensing techniques but the idea of the Direction of Arrival of the signal when the signal is sensed is equally important. ESPRIT is a high-resolution signal parameter estimation technique based on the translational invariance structure of a sensor array. The ESPRIT algorithm is an attractive solution to many parameter estimation problems due to its low computational cost. The Estimation of DOA of three signals is investigated in this paper.
\end{abstract}

\section{Keywords}

Cognitive Radio, Direction of Arrival, Beam forming, Subarray,ESPRIT.

\section{INTRODUCTION}

Smart antenna are the antenna arrays with smart signal processing algorithms used to identify spatial signal signature such as direction of arrival (DOA) of the signal. Smart antenna system estimates the DOA using techniques such as MUSIC (Multiple Signal Classification), ESPRIT (Estimation of signal parameters via rotational via rotational invariance). EPRIT overcomes problems with a dramatic reduction in computational and storage requirements by exploiting a property called the shift invariance of the array, Unlike most DOA estimation methods such as MUSIC, ESPRIT does not require that the array manifold steering vectors be precisely known [2], so the array calibration requirements are not stringent. The ESPRIT algorithm is applicable to a particular class of sensor arrays whose geometry is shift invariant. Computation complexity and storage requirements are lower than MUSIC as it does not involve extensive search throughout all possible steering vectors [3].

\section{ESPRIT ALGORITHM}

ESPRIT achieves a reduction in computational complexity by imposing a constraint on the structure of an array. The ESPRIT algorithm assumes that an antenna array is composed of two identical subarrays (see Figure). The subarrays may overlap, that is, an array element may be a member of both subarrays.

If there are a total of $\mathrm{M}$ elements in an array and $\mathrm{m}$ elements in each subarray, the overlap implies that $\mathrm{M} \leq 2 \mathrm{~m}$. For subarrays that do not overlap, $\mathrm{M}=2 \mathrm{~m}$. The individual elements of each subarray can have arbitrary polarization, directional gain, and phase response, provided that each has an identical twin in its companion subarray[5][6]. Elements of each pair of identical sensors, or doublet, are assumed to be separated physically by a fixed displacement (translational) vector. The array thus, displacement vectors). This property leads to the rotational invariance of signal subspaces spanned by the data vectors associated with the spatially displaced subarrays; the invariance is then utilized by ESPRIT to find DOAs.

\author{
Rabindranath Bera \\ Dept. of Electronics and Communication Sikkim \\ Manipal Institute of Technology
}

Assume $d$ signals impinging onto the array. Let $x 1(t)$ and $\mathrm{x} 2(\mathrm{t})$ represent the signal received by the two subarrays corrupted by additive noise $n 1(t)$ and $n 2(t)$. Each of the subarrays has $m$ elements., the signals received can then be expressed as

$$
\begin{aligned}
& \mathrm{X} 1(\mathrm{t})=[\mathrm{a}(\mu 1), . ., \mathrm{a}(\mu \mathrm{d})] \mathrm{s}(\mathrm{t})+\mathrm{n} 1(\mathrm{t}) \\
& =\mathrm{As}(\mathrm{t})+\mathrm{n} 1(\mathrm{t}) \\
& \mathrm{X} 2(\mathrm{t})=[\mathrm{a}(\mu 1) \operatorname{ej} \mu 1, . ., \mathrm{a}(\mu \mathrm{d}) \text { ej } \mu \mathrm{d}] \mathrm{s}(\mathrm{t})+\mathrm{n} 2(\mathrm{t}) \\
& =\mathrm{A} \Phi \mathrm{s}(\mathrm{t})+\mathrm{n} 2(\mathrm{t})
\end{aligned}
$$

where $\mathrm{x}_{1}(\mathrm{t})$ and $\mathrm{x}_{2}(\mathrm{t})$ are the $\mathrm{m} * 1$ vectors representing the data received by the first and second subarrays, respectively. $\mathrm{n}_{1}(\mathrm{t})$ and $\mathrm{n}_{2}(\mathrm{t})$ are the $\mathrm{m} * 1$ vectors representing the noises received by the two subarrays, respectively. $A=\left[a\left(\mu_{1}\right), \ldots\right.$, $\left.\mathrm{a}\left(\mu_{\mathrm{d}}\right)\right]$ is the $\mathrm{m} * \mathrm{~d}$ steering matrix of the subarray. $\mathrm{s}(\mathrm{t})$ is the signals received by the first subarray [3].

$\Phi=\operatorname{diag}\left[e j \mu_{1}, . .\right.$, ej $\left.\mu_{\mathrm{d}}\right]$ is a $\mathrm{d} * \mathrm{~d}$ diagonal matrix that relates the signals received by the two subarrays and is called the rotation operator. It is caused by the fact that the signals arriving at the second subarray will experience an extra delay due to the fixed displacement $\Delta$ between the two subarrays.

Given $\mathrm{N}$ snapshots, $\mathrm{x}(\mathrm{t} 1), \mathrm{x}(\mathrm{t} 2), \ldots, \mathrm{x}(\mathrm{tn})$, the objective of the ESPRIT technique is to estimate the DOAs via an estimation of $\mu \mathrm{i}$ by Determining $\Phi=\operatorname{diag}[\operatorname{ej} \mu 1, \ldots$, ej $\mu \mathrm{d}]$ In doing so, two steps are required based on the data received by the array: estimating the signal subspace and then estimating the subspace rotation operator. we take the uniform linear array (ULAs) as an example to illustrate the standard ESPRIT procedure. Consider a ULA consisting of M elements.

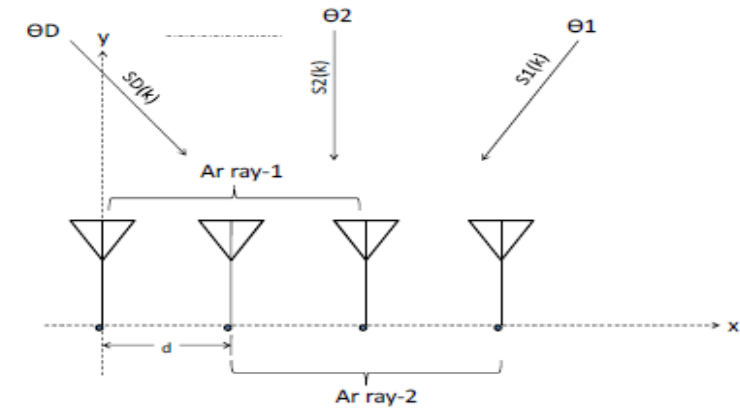

Fig.1 Four elements linear array with two doublets.

The two subarrays, array- 1 and array- 2 are displaced by distance ' $d$ '. The signals induced on each of the arrays are given by

$$
\mathrm{x} 1(\mathrm{k})=\mathrm{A} 1 *_{\mathrm{s}}(\mathrm{k})+\mathrm{n} 1(\mathrm{k})
$$

and

$$
\mathrm{x} 2(\mathrm{k})=\mathrm{A} 1 * \Lambda * \mathrm{~s}(\mathrm{k})+\mathrm{n} 2(\mathrm{k})
$$




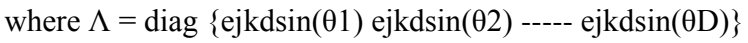

$=\mathrm{D} \times \mathrm{D}$ diagonal unitary matrix with phase shifts between doublets for each DOA.

Creating the signal subspace for the two subarrays results in two matrices V1 \& V2. Since the arrays are translationally related, the subspaces of eigenvectors are related by a unique nonsingular transformation matrix $\phi$ such that [4]

$$
\mathrm{V} 1 \phi=\mathrm{V} 2
$$

There must also exist a unique nonsingular transformation matrix $\mathrm{T}$ such that

$$
\mathrm{V} 1=\mathrm{AT} \text { and } \mathrm{V} 2=\mathrm{A} \Lambda \mathrm{T}
$$

and finally we can derive

$$
\mathrm{T} \Phi \mathrm{T}-1=\Lambda
$$

Thus the eigenvalues of $\phi$ must be equal to the diagonal elements of $\Lambda$ such that

$\lambda 1=e j k d \sin (\theta 1), \lambda 2=e j k d \sin (\theta 2)-----\lambda D=e j k d \sin (\theta \mathrm{D})$

Once the eigenvalues of $\phi, \lambda 1, \lambda 2,-----\lambda D$ are calculated, we can estimate the angles of arrivals as

$$
\theta \mathrm{i}=\sin -1(\arg (\lambda \mathrm{i}) / \mathrm{kd})
$$

ESPRIT eliminates the search procedure \& produces the DOA estimation directly in terms of the eigenvalues without much computational and storage requirements. The ESPRIT method estimates signal DOA by finding the roots of two independent

equations closest to the unit circle. This method does not require using a scan vector to scan over all possible directions like the MUSIC (Multiple Signal Classification) algorithm.

\section{SIMULATION RESULT}

In the block diagram, three constant data are transmitteding in order to check the proper data transmission along with DOA estimation.

The three complex signals have the theta value as 30,20 and -10. 2D Rectangular Array Tx Digital Beam Synthesis is used to set the theta values. For the transmission and reception $8 \times 8$ antenna is used. The Mathlang block consist the ESPRIT algorithm to find the arrival angle of each signal.

The 16 antenna elements are considered with carrier frequency of $10 \mathrm{GHz}$. The graph below showing the detection of three signals having different angle of arrival.

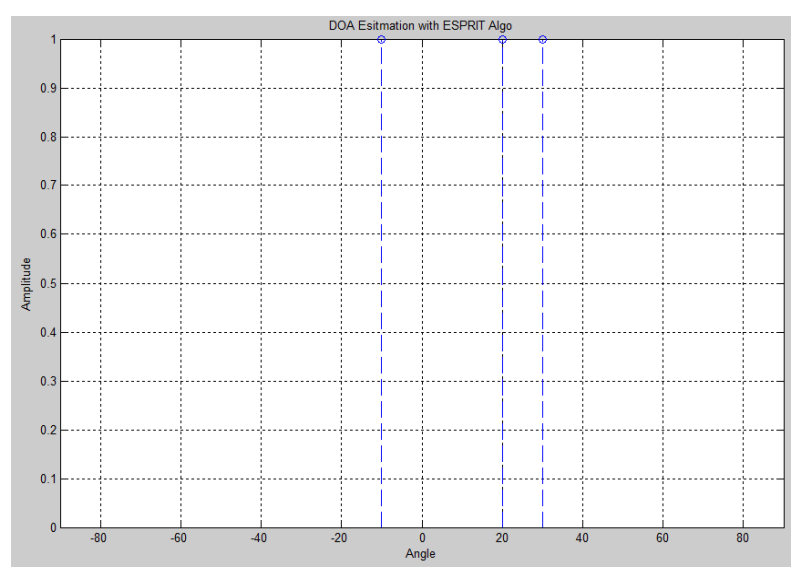

Fig. 3 Detection of three signals at $-10,20$ and 30 .

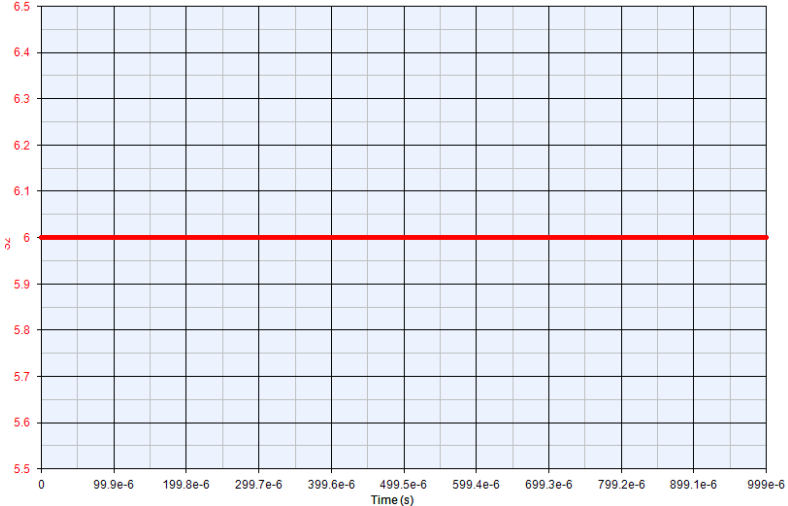

Fig. 4 Transmitted Data at 30 degree.

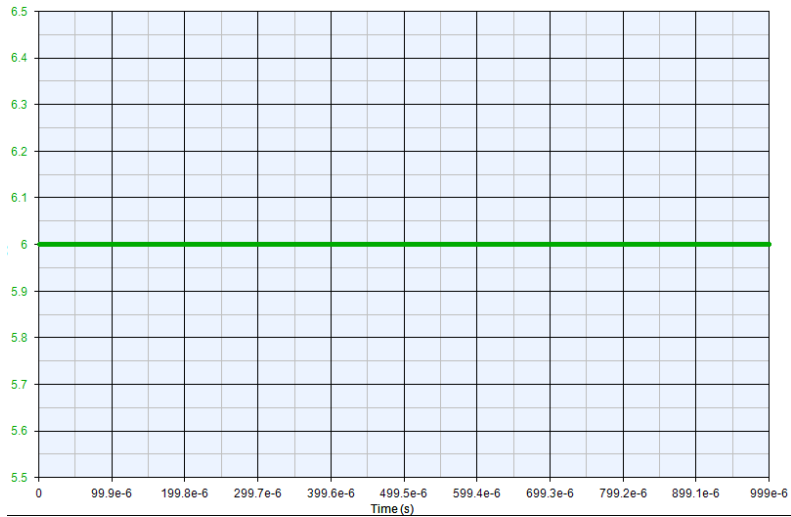

Fig. 5 Received Data at 30 degree.

As we can clearly see the data we transmitted at 30 degree is exactly receiving at receiver.

\section{CONCLUSION}

This paper shows the algorithm has successfully estimated the Direction of Arrival of the incidents signals received on the antenna array. ESPRIT exploits such a displacement property which translates into an underlying rotational invariance of signal subspaces spanned by two data vectors received by two subarrays. The simulation results show that ESPRIT Algorithm estimated the DOA of signals.

With the increase in noise we do not achieve precise values of the angle of arrival. So use of attenuation of noise signal in the practical implementation is to be carried out. Also effect of noise along with multipath fading also can be seen. Smart antennas concept is being used in this project. But smart antenna does not have intelligence. So some learning Algorithms having some intelligence can be seen.

\section{REFERENCES}

[1] Jose Bermudez, Radwan C.Chin, "Simulation Study on DOA Estimation using ESPRIT Algorithm" Processing of the World Congress on Engineering and Computer Science 2009 Vol I, October 20-22,2009, San Francisco, USA.

[2] Ding, W. and Marchionini, G. 1997 A Study on Video Browsing Strategies. Technical Report. University of Maryland at College Park. 
[3] R.O Schmidt, "Multiple Emitter location and Signal Parameter Estimation, "IEEE Trans. Antennas propagation, Vol AP-34

[4] Forman, G. 2003. An extensive empirical study of feature selection metrics for text classification. J. Mach. Learn. Res. 3 (Mar. 2003), 1289-1305.

[5] Y.T. Yu, M.F. Lau, "A comparison of MC/DC, MUMCUT and several other coverage criteria for logical decisions", Journal of Systems and Software, 2005, in press.
[6] Chetan R.Dongarsane, A.N.Jadhav and Swapnil M.Hirikude "Performance Analysis of ESPRIT Algorithm for Smart Antenna System" International Journal of Communication Network and Security (IJCNS), Vol-1, Issue-3 ISSN: 2231-1882

[7] Lal. C. Godara. "Limitations and capabilities of directions of arrival estimation techniques using an array of antennas: A Mobile communications perspective, "proceeding of IEEE, pp 327-333,1996.

[8] Frank Gross, "Smart antennas for wireless communications," McGraw Hill. New York, 2005 